\title{
Mycophenolic acid in the treatment of birdshot chorioretinopathy: long-term follow-up
}

\author{
Deshka Doycheva, ${ }^{1}$ Herbert Jägle, ${ }^{1,2}$ Manfred Zierhut, ${ }^{1}$ Christoph Deuter, ${ }^{1}$ \\ Gunnar Blumenstock, ${ }^{3}$ Ulrich Schiefer, ${ }^{1,4}$ Katarina Stingl, ${ }^{1}$ Kai Januschowski, ${ }^{1}$ \\ Bogomil Voykov, ${ }^{1}$ Nicole Stuebiger ${ }^{5}$
}

${ }^{1}$ Centre for Ophthalmology, University of Tuebingen, Tuebingen, Germany

${ }^{2}$ Department of Ophthalmology, University of Regensburg, Regensburg, Germany

${ }^{3}$ Department of Clinical Epidemiology and Applied Biometry, University of Tuebingen, Tuebingen, Germany

${ }^{4}$ Competence Center "Vision Research", Study Course

"Ophthalmic Optics/ Audiology", University of Applied Sciences, Aalen, Germany

Department of Ophthalmology, Charité, University Medicine Berlin, Berlin, Germany

\section{Correspondence to} Dr Deshka Doycheva, Centre for Ophthalmology, University of Tuebingen, Schleichstr. 12, Tuebingen 72076, Germany; deshka.doycheva@med.unituebingen.de

Received 13 May 2014 Revised 2 July 2014 Accepted 13 July 2014 Published Online First 24 July 2014
CrossMark

To cite: Doycheva $D$, Jägle $\mathrm{H}$, Zierhut $\mathrm{M}$, et al. $\mathrm{Br}$ J Ophthalmol 2015;99: 87-91.

\section{ABSTRACT}

Aim To assess the long-term efficacy and tolerability of both derivatives of mycophenolic acid, mycophenolate mofetil (MMF) and mycophenolate sodium (MPS), in the therapy of patients with birdshot chorioretinopathy (BSCR).

Methods Retrospective analysis of 24 patients (48 eyes) with BSCR, treated with MMF or MPS with a follow-up of at least 1 year. The main outcome measures included control of inflammation, steroid-sparing potential and side effects. Secondary outcome measure was the development of retinal function during the therapy measured by best-corrected visual acuity (BCVA), visual field and/or electroretinography (ERG).

Results Twelve patients (50\%) were treated with MMF and 12 patients (50\%) with MPS. Control of intraocular inflammation, defined as complete lack of clinical and angiographic signs of inflammatory activity, was achieved in 16 of 24 patients $(67 \%)$. The angiographic signs of activity were significantly reduced during the follow-up $(p<0.05)$. No significant difference was found in the mean BCVA, the visual field and the ERG parameters during the treatment compared with the baseline ( $p>0.05$ ). In 20 out of 21 patients (95\%) who received systemic corticosteroids, the corticosteroids could be tapered to a daily dose of $\leq 10 \mathrm{mg}$ (rate $0.26 /$ patientyear). Drug-related side effects occurred in 12 patients (50\%, rate $0.16 /$ patient-year). In four patients (17\%), a therapy switch from MMF to MPS was undertaken due to gastrointestinal discomfort.

Conclusions Derivatives of mycophenolic acid are effective and safe drugs for the treatment of BSCR. In cases with gastrointestinal side effects, a therapy switch from MMF to MPS should be considered.

\section{INTRODUCTION}

Birdshot chorioretinopathy (BSCR) is a form of chronic bilateral posterior uveitis with progressive course and poor long-term visual prognosis. ${ }^{1}$ Characteristic for the disease is the presence of 'birdshot lesions', manifesting as hypopigmented cream-coloured round or oval choroidal lesions, localised around the optic disc. ${ }^{2}$ The disease is strongly associated with the human leucocyte antigen (HLA)-A29. ${ }^{3}$ Due to its chronic relentless course, BSCR may lead to severe visual impairment and even blindness. ${ }^{1}$ Because of the poor visual outcome during corticosteroid monotherapy, early treatment with immunosuppressive agents has been recommended for the management of patients with BSCR. $^{5-7}$
Mycophenolic acid (MPA) is an immunosuppressive agent that selectively inhibits the proliferation of human T-lymphocytes and B-lymphocytes. ${ }^{8}$ Both derivatives of MPA, mycophenolate mofetil (MMF) and mycophenolate sodium (MPS), have been shown to be effective for the therapy of chronic non-infectious uveitis. ${ }^{9-13}$ To date, only limited data are available about the use of MMF as a steroidsparing agent in the treatment of BSCR. ${ }^{71415}$ To the best of our knowledge, the use of MPS in the treatment of BSCR has not been previously reported. The aim of this study was to assess the long-term efficacy and tolerability of both derivatives of MPA, MMF and MPS, in the therapy of BSCR.

\section{METHODS \\ Patients}

A retrospective analysis was performed including all patients with BSCR who were treated at our institution with MMF or MPS and who had a follow-up of at least 1 year. BSCR was diagnosed according to the criteria established by an international consensus panel. ${ }^{2}$ In all patients, an extensive uveitis work-up was performed to exclude other diseases that can cause multifocal choroidal lesions. Additionally, HLA-A29 typing was performed in all patients.

A full ophthalmological examination, including the assessment of best-corrected visual acuity (BCVA), tonometry, slit-lamp biomicroscopy and fundus exam, was performed in all patients. Additional ophthalmological investigations including fluorescein angiography (FA), optical coherence tomography (OCT), visual field and electroretinography (ERG) were performed in most patients.

\section{Treatment}

MMF was administered at a dose of $1000 \mathrm{mg}$ twice daily, defined as a standard dosage for MMF. MPS was given at a dose of $720 \mathrm{mg}$ twice daily, defined as a standard dosage for MPS. Depending on the activity of intraocular inflammation, systemic prednisolone in an initial dosage of $1 \mathrm{mg} / \mathrm{kg} /$ days was administered in 21 patients. Prednisolone was gradually tapered on a weekly basis (in $10 \mathrm{mg}$ steps to $30 \mathrm{mg} /$ days, in $5 \mathrm{mg}$ steps to $20 \mathrm{mg} /$ days, followed by $2.5 \mathrm{mg}$ steps) to a maintenance dose of $\leq 10 \mathrm{mg}$ daily.

\section{Outcome measures}

The main outcome measures of the study were (i) control of inflammation, (ii) steroid-sparing 
potential and (iii) long-term side effects during MMF/MPS treatment. The inflammatory cell grade was assessed according to the recommendations of Nussenblatt et $a l^{16}$ and the Standardization of Uveitis Nomenclature Working Group (SUN). ${ }^{17}$ FA was additionally used to assess disease activity. Angiographic signs in active disease included hyperfluorescence of the optic disc and retinal vascular leakage. ${ }^{7}$ The optic disc hyperfluorescence and retinal vascular leakage were graded as absent (0), mild (1) and strong (2). The presence of cystoid macular oedema (CMO) was detected by ophthalmoscopy, OCT or FA. Control of intraocular inflammation was defined as complete lack of clinical and angiographic signs of inflammatory activity. A relapse of BSCR was considered in case of active fundus lesions, an increase of cell grade or angiographic signs of activity. The MMF/MPS treatment was assessed as ineffective if one or more of the following findings were observed: persistent intraocular inflammation, refractive $\mathrm{CMO}$ or development of CMO for the first time during therapy. Corticosteroid-sparing success was defined as inactive inflammation after tapering of prednisolone to $\leq 10 \mathrm{mg}$ daily, as recommended by the SUN Working Group. ${ }^{17}$

The patients were followed up every 3 months. Treatment-related side effects were assessed in each patient at each visit by targeted patient history regarding subjective symptoms of side effects and monthly laboratory investigations, performed by general physicians, including complete blood count with differential, liver enzymes and kidney functional tests.

The secondary outcome measure was the development of the retinal function during the MMF/MPS therapy. Retinal function was measured by BCVA, visual field or full-field ERG. BCVA was assessed in European decimals and converted to logarithm of minimum angle of resolution ( $\log M A R)$ for statistical analysis. According to the SUN recommendations, improvement/ worsening of BCVA was defined as a change (decrease/increase) of at least $0.3 \log$ MAR (three lines). ${ }^{17}$ Visual field testing was performed using automated static perimetry within the central $30^{\circ}$ visual field (Tuebingen Automatic Perimeter, TAP, Oculus Inc., Germany; or Octopus 101 perimeter, Haag-Streit Inc, Switzerland). The following data were collected for each visual field test: foveal threshold (ie, central differential luminance sensitivity) in decibels $(\mathrm{dB})$, and occurrence and pattern of visual field defects within the central $30^{\circ}$ visual field. Change in the foveal threshold during the treatment in comparison to the therapy start (baseline) was considered if the foveal threshold increased or decreased by $\geq 5 \mathrm{~dB}$. The scotoma extent within the central $30^{\circ}$ visual field eccentricity was estimated by counting the locations with absolute or relative visual field defects. Change was considered if a difference of at least $10 \%$ was found in the number of test locations with absolute or relative visual field loss between baseline and follow-up. Full-field ERGs were recorded using DTL fibre electrodes with Espion $\mathrm{E}^{2}$ (Diagnosys LLC) and a ColorDome (Diagnosys LLC) according to the standards of the International Society for Clinical Electrophysiology of Vision (ISCEV). ${ }^{18}$ For this study, amplitudes and implicit times of the dark-adapted 3.0 ERG (combined rod-cone responses) and the light-adapted $30 \mathrm{~Hz}$ flicker responses were evaluated. Change was considered if a difference of at least $15 \%$ was observed in the amplitudes or implicit times between baseline and follow-up visits.

\section{Statistical methods}

The incidence rate per patient-year (PY) was calculated as the number of events divided by the amount of patient-time at risk. Statistically significant differences were evaluated using the
Table 1 Baseline characteristics of 24 patients with birdshot chorioretinopathy treated with derivatives of mycophenolic acid

\begin{tabular}{lc}
\hline Patient characteristics & $\begin{array}{c}\text { Number of patients } \\
\text { or period of time }\end{array}$ \\
\hline Mean age at therapy start, (median, range) [years] & $51.5(54.5,30-71)$ \\
Gender, female [n/N (\%)] & $14 / 24(58)$ \\
Bilaterality [n/N (\%)] & $24 / 24(100)$ \\
Mean duration of disease at therapy start, (median, & $29.2(20,5-115)$ \\
range) [months] & \\
HLA-A29 positive [n/N (\%)] & $24 / 24(100)$ \\
Previous treatment [n/N (\%)] & $14 / 24(58)$ \\
CS monotherapy & $11 / 24(46)$ \\
1 IS agent (CSA) & $1 / 24(4)$ \\
CS+1 IS agent (AZA, MTX) & $2 / 24(8)$ \\
Reason for initiation of MMF/MPS therapy [n/N (\%)] & \\
Activity during CS treatment & $5 / 24(21)$ \\
Activity during previous IS treatment & $2 / 24(8)$ \\
Side effects of previous treatment & $1 / 24(4)$ \\
MMF/MPS as a first IS agent & $16 / 24(67)$ \\
Mean follow-up time (median, range) [months] & $43(33,12-106)$ \\
Mean duration of MMF/MPS therapy & $38(32,12-79)$ \\
(median, range) [months] & \\
\hline AZA, azathioprine; CS, corticosteroids; CSA, cyclosporine A; IS, immunosuppressive \\
agent; MMF, mycophenolate mofetil; MPS, mycophenolate sodium; MTX, \\
methotrexate; $n$, number of affected patients; $N$, total number of patients.
\end{tabular}

Fisher's exact test, sign test, Wilcoxon signed-rank test and paired $t$ test, as appropriate. $p$ Values less than 0.05 were considered to be statistically significant. The performed data analysis was explanatory, and the multiple significance tests were applied only for descriptive purposes.

This work adhered to the tenets of the Declaration of Helsinki, and Institutional Ethics Committee of University of Tuebingen granted approval with waiver of informed consent for this retrospective study.

\section{RESULTS}

\section{Characteristics of the study population}

Twenty-four patients (48 eyes) with BSCR, who were treated with MMF or MPS and who had a follow-up of at least 1 year, were included in the study. All patients had bilateral disease and were HLA-A29 positive. In 12 patients (50\%), the treatment was started with MMF and in 12 patients (50\%) with MPS. The mean follow-up after therapy initiation was 43 months (median 33 months, range 12-106 months). Baseline patient characteristics, previous treatment and reason for starting mycophenolate therapy are presented in table 1 .

\section{Treatment outcome}

\section{Control of intraocular inflammation}

Control of intraocular inflammation was achieved in 16 of 24 patients $(67 \%)$ during the treatment with standard dosage of MMF or MPS. In five of these patients, a relapse of BSCR occurred during the mean treatment duration of 35 months (median 33 months, range $28-42$ months). In 8 of the 24 patients $(33 \%)$, the treatment was assessed as ineffective due to inflammatory activity ( 2 patients), inflammatory activity associated with development of CMO (3 patients), new-onset CMO (1 patient) or persistent CMO during the therapy (2 patients). The mean relapse rate in the entire group of 24 patients was 0.2 relapses per PY (95\% CI 0.11 to 0.33$)$. 
We separately analysed the efficacy of each of both derivatives of MPA. Control of intraocular inflammation was achieved in 9 of the 12 patients $(75 \%)$ treated with MMF and in 7 of the 12 patients $(58 \%)$ treated with MPS, respectively (Fisher's exact test, $\mathrm{p}=0.67$ ). MMF was assessed as ineffective in three patients (25\%) because of inflammatory activity (two patients) or inflammatory activity with CMO (one patient). MPS was assessed as ineffective in five patients (42\%) due to inflammatory activity with CMO (two patients), new-onset CMO (one patient) or persistent CMO during the therapy (two patients).

At baseline, CMO was present in seven patients (29\%). During the follow-up, complete resolution of $\mathrm{CMO}$ was observed in five patients and partial resolution in two patients. CMO occurred for the first time during the MMF/MPS treatment in four patients (17\%, rate $0.05 / \mathrm{PY})$.

FA was performed in 22 patients. The angiographic signs of activity were significantly reduced during the follow-up $(\mathrm{p}<0.05)$. FA data are presented in detail in table 2 .

\section{Steroid-sparing effect}

During the treatment period, 21 patients $(88 \%)$ received systemic corticosteroids. In 20 of these patients (95\%), the corticosteroids could be tapered to a daily dose of $\leq 10 \mathrm{mg}$ (rate $0.26 / \mathrm{PY}$ ). Corticosteroid tapering to a maintenance dose of $5 \mathrm{mg}$ daily could be achieved in 12 patients $(57 \%$, rate 0.16 / PY). Systemic corticosteroids were discontinued in three patients (14\%, rate $0.04 / \mathrm{PY})$.

Table 2 Fluorescein angiographic parameters in 24 patients with birdshot chorioretinopathy treated with derivatives of mycophenolic acid

\begin{tabular}{|c|c|c|c|c|}
\hline FA parameters & Baseline & After 1 year & After 2 years & Last visit \\
\hline \multicolumn{5}{|c|}{ Optic disc hyperfluorescence [n/N eyes] } \\
\hline \multicolumn{5}{|l|}{ Right eyes } \\
\hline No & $1 / 22$ & $0 / 13$ & $2 / 16$ & $3 / 21$ \\
\hline Mild & $4 / 22$ & $8 / 13$ & $12 / 16$ & $14 / 21$ \\
\hline Strong & $17 / 22$ & $5 / 13$ & $2 / 16$ & $4 / 21$ \\
\hline \multicolumn{5}{|l|}{ Left eyes } \\
\hline No & $1 / 22$ & $0 / 13$ & $1 / 16$ & $2 / 21$ \\
\hline Mild & $4 / 22$ & $8 / 13$ & $13 / 16$ & $15 / 21$ \\
\hline Strong & $17 / 22$ & $5 / 13$ & $2 / 16$ & $4 / 21$ \\
\hline \multicolumn{5}{|c|}{ Comparison with baseline, $p$ value* } \\
\hline Right eyes & N/A & 0.29 & $0.012 \dagger$ & $<0.001 \dagger$ \\
\hline Left eyes & N/A & 0.29 & $0.012 \dagger$ & $<0.001 \dagger$ \\
\hline \multicolumn{5}{|c|}{ Retinal vascular leakage [n/N eyes] } \\
\hline \multicolumn{5}{|c|}{ Right eyes } \\
\hline No & $4 / 22$ & $4 / 13$ & $8 / 16$ & $12 / 21$ \\
\hline Mild & $4 / 22$ & $6 / 13$ & $8 / 16$ & $8 / 21$ \\
\hline Strong & $14 / 22$ & $3 / 13$ & $0 / 16$ & $1 / 21$ \\
\hline \multicolumn{5}{|l|}{ Left eyes } \\
\hline No & $3 / 22$ & $4 / 13$ & $7 / 16$ & $12 / 21$ \\
\hline Mild & $4 / 22$ & $6 / 13$ & $9 / 16$ & $8 / 21$ \\
\hline Strong & $15 / 22$ & $3 / 13$ & $0 / 16$ & $1 / 21$ \\
\hline \multicolumn{5}{|c|}{ Comparison with baseline, $p$ value ${ }^{*}$} \\
\hline Right eyes & $\mathrm{N} / \mathrm{A}$ & 0.07 & $0.022 \dagger$ & $0.002 \dagger$ \\
\hline Left eyes & $N / A$ & 0.07 & $0.013+$ & $0.001 \dagger$ \\
\hline
\end{tabular}

\section{Development of retinal function}

Best-corrected visual acuity

Mean $\log$ MAR BCVA did not statistically significantly differ from baseline over the follow-up period ( $p>0.05$, table 3$)$. At baseline, BCVA of $\leq 20 / 40$ (logMAR 0.3 ) in the worse eye was found in 12 patients $(50 \%)$, in 3 patients $(13 \%)$ it was $\leq 20 / 200$ $(\log$ MAR 1$)$. At the end of follow-up, BCVA of $\leq 20 / 40$ (logMAR 0.3 ) in the worse eye was observed in 10 patients $(42 \%)$, in 2 patients $(8 \%)$ it was $\leq 20 / 200(\log$ MAR 1$)$.

Visual field

A visual field examination was performed in 22 patients. The common observed visual field patterns were enlarged blind spot (20\% of eyes), disseminated visual field defects (30\%), central and paracentral scotoma (18\%) and arcuate visual field defects (14\%). In $16 \%$ of eyes, no visual field defects were found. In general, the foveal threshold and the number of absolute and relative visual field defects within the central $30^{\circ}$ visual field did not differ significantly between baseline and follow-up visits (table 3).

\section{Electroretinography}

Full-field ERG was performed in 21 patients. None of the analysed ERG parameters, including amplitudes and implicit times of the combined rod-cone responses and the light-adapted $30 \mathrm{~Hz}$ responses, showed statistically significant differences over the treatment period ( $p>0.05$; data not shown).

\section{Therapy change}

The mean duration of MMF/MPS treatment was 38 months (median 32 months, range 12-79 months). At the end of follow-up, 15 patients (63\%) received MMF or MPS as the only corticosteroid-sparing agent. In three patients (8\%, rate 0.03 / PY), the treatment could be discontinued due to control of inflammation. Therapy change was necessary in seven patients (29\%, rate 0.09/PY): four of these patients received interferon- $\alpha$ in addition due to refractory CMO, methotrexate was added to MMF in two patients due to activity of intraocular inflammation and the therapy was switched to adalimumab in one patient due to inflammatory activity with CMO.

\section{Side effects}

Drug-related side effects occurred in 12 patients (50\%, rate $0.16 / \mathrm{PY})$. Of these, five patients experienced more than one adverse effect. The rate of side effects during the treatment was $0.24 / \mathrm{PY}$. The most common side effects were gastrointestinal discomfort $(n=5)$, increased liver enzymes $(n=3)$, headache and malaise $(n=2)$, susceptibility to infection $(n=2)$ and skin rash $(n=2)$. In six patients, a reduction of MMF/MPS was required due to adverse effects (25\%, rate $0.08 / \mathrm{PY})$. In four patients (17\%, rate $0.05 / \mathrm{PY})$, the therapy was switched from MMF to MPS due to gastrointestinal discomfort. In all four patients, the gastrointestinal side effects disappeared during MPS treatment. No severe adverse effects requiring therapy discontinuation were observed.

\section{DISCUSSION}

The treatment of BSCR is a challenge due to its chronic progressive course leading to severe visual impairment. To achieve inflammatory control and preserve vision, early steroid-sparing treatment is recommended. ${ }^{5} 719$ In this study, we report on the long-term results with both derivatives of MPA, MMF and MPS, in the treatment of patients with BSCR. We achieved 
Table 3 Best-corrected visual acuity and visual field parameters at baseline and follow-up in 24 patients with birdshot chorioretinopathy treated with derivatives of mycophenolic acid

\begin{tabular}{|c|c|c|c|c|}
\hline Parameters & Baseline & After 1 year & After 2 years & Last visit \\
\hline \multicolumn{5}{|l|}{ BCVA [logMAR] } \\
\hline Right eyes: mean $( \pm S D)$ & $0.25( \pm 0.25)$ & $0.24( \pm 0.38)$ & $0.29( \pm 0.39)$ & $0.19( \pm 0.31)$ \\
\hline Left eyes: mean $( \pm$ SD) & $0.32( \pm 0.41)$ & $0.37( \pm 0.51)$ & $0.34( \pm 0.44)$ & $0.3( \pm 0.44)$ \\
\hline \multicolumn{5}{|c|}{ Comparison with baseline, $p$ value* } \\
\hline Right eyes & N/A & 0.837 & 1.0 & 0.245 \\
\hline Left eyes & N/A & 0.446 & 0.452 & 0.835 \\
\hline \multicolumn{5}{|l|}{ Foveal threshold [dB] } \\
\hline Right eyes: mean $( \pm S D)$ & $27.2( \pm 6.7)$ & $29.9( \pm 4.6)$ & $29.7( \pm 3.8)$ & $28.5( \pm 5.9)$ \\
\hline Left eyes: mean $( \pm S D)$ & $25.8( \pm 8.5)$ & $27.4( \pm 4.9)$ & $26.5( \pm 7.8)$ & $26.4( \pm 6.6)$ \\
\hline \multicolumn{5}{|c|}{ Comparison with baseline, $p$ value* } \\
\hline Right eyes & $\mathrm{N} / \mathrm{A}$ & 0.560 & 0.367 & 0.370 \\
\hline Left eyes & N/A & 0.326 & 0.597 & 0.908 \\
\hline \multicolumn{5}{|c|}{ No. of absolute VFDs within the central $30^{\circ}$ visual field [n] } \\
\hline Right eyes: median (IQR) & $1.5(0-4)$ & $2(0-12)$ & $2(0-5)$ & $3(0-10)$ \\
\hline Left eyes: median (IQR) & $4(0-14.5)$ & $2.5(0.3-8.5)$ & $6(2-10)$ & $4(2-7)$ \\
\hline \multicolumn{5}{|c|}{ Comparison with baseline, $p$ valuet } \\
\hline Right eyes & N/A & 0.173 & 1.0 & 0.143 \\
\hline Left eyes & N/A & 0.730 & 0.611 & 0.937 \\
\hline \multicolumn{5}{|c|}{ No. of relative VFDs within the central $30^{\circ}$ visual field [n] } \\
\hline Right eyes: median (IQR) & $12(4.8-38.8)$ & $9(6.3-12.8)$ & $9(4-16)$ & $7(4-19)$ \\
\hline Left eyes: median (IQR) & $29(4-38.3)$ & $7(3.3-24.8)$ & $16(5-26)$ & $16(5-27)$ \\
\hline \multicolumn{5}{|c|}{ Comparison with baseline, $p$ valuet } \\
\hline Right eyes & N/A & 0.126 & 0.068 & $0.026 \ddagger$ \\
\hline Left eyes & N/A & 0.906 & 0.327 & 0.648 \\
\hline
\end{tabular}

control of inflammation in $67 \%$ of the patients treated with MMF or MPS. Some previous studies have reported the use of various immunosuppressive agents, including MMF, in patients with BSCR. ${ }^{6} 7{ }^{15}$ However, the outcome of MMF treatment was not analysed separately from the other immunosuppressive drugs. Recently, Cervantes-Castaneda et $a l^{20}$ presented their results of combined immunosuppressive therapy with cyclosporine $\mathrm{A}$ and MMF in patients with BSCR. The authors reported on the control of inflammation in $67.4 \%$ of the patients. However, therapy discontinuation because of side effects was necessary in $10 \%$ of the patients. Because of differences in the therapy schemata and study aims, the study of Cervantes-Castaneda et al and our study are not fully comparable. Interestingly, we observed a similar efficacy of the BSCR treatment with only one steroid-sparing agent (MMF or MPS). Moreover, no severe side effects requiring therapy discontinuation were found in our patients. As a combined therapy with two immunosuppressive agents may be associated with an increased risk of malignancies and organ toxicity, we prefer to initiate treatment with just one steroid-sparing drug. In the case of inefficacy, therapy with biological agents may be considered.

When analysing the efficacy of MMF and MPS separately, no significant difference in the anti-inflammatory potency of both MPA derivatives was observed. However, in $17 \%$ of the patients, a therapy switch from MMF to MPS was needed because of gastrointestinal side effects. Gastrointestinal discomfort is the most common side effect of MMF, and the necessity of dose reduction or even discontinuation of the drug may limit its clinical benefit. ${ }^{9} 1021$ In such cases, MPS can be used due to its superior gastrointestinal side effect profile. A therapy switch from MMF to MPS has been previously reported in patients with various inflammatory eye diseases. ${ }^{11} 12$ Our observation shows that in cases of adverse effects during MMF treatment, a switch to MPS is also an option in patients with BSCR.

CMO is the most common complication of BSCR and has been found in up to $84 \%$ of the patients suffering from this disease. ${ }^{1}$ In our study, we observed a lower incidence of CMO indicating that the derivatives of MPA have the potential to reduce the risk of $\mathrm{CMO}$. However, they cannot completely prevent the occurrence of CMO. A similar observation has been reported by our own group in patients with chronic noninfectious uveitis treated with MMF. ${ }^{22}$

With regards to functional assessment, it is well known that in BSCR, exclusive BCVA assessment should not be considered as a reliable parameter for evaluating therapy response. ${ }^{7202324}$ In the present study, BCVA, visual field and full-field ERG parameters remained stable during the treatment and no worsening of the retinal function was observed. This suggests that MMF and MPS are effective not only in controlling inflammation but also in the long-term preservation of retinal function.

We are aware of the limitations of our study arising from its retrospective nature. However, this is the first study that has assessed the efficacy of both derivatives of MPA, MMF and MPS, in the treatment of BSCR according to standardised criteria.

In conclusion, MMF and MPS were shown to be effective and safe steroid-sparing agents in the therapy of BSCR, and we recommend them as a first-line immunosuppressive treatment in 
this severe sight-threatening disease. In case of gastrointestinal side effects during MMF treatment, a therapy switch to MPS should be performed because of its better gastrointestinal tolerability.

\section{Competing interests None.}

Ethics approval Institutional Ethics Committee of University of Tuebingen, Germany.

Provenance and peer review Not commissioned; externally peer reviewed.

\section{REFERENCES}

1 Rothova A, Berendschot TT, Probst K, et al. Birdshot corioretinopathy: long-term manifestations and visual prognosis. Ophthalmology 2004;111:954-9.

2 Levinson RD, Brezin A, Rothova A, et al. Research criteria for the diagnosis of birdshot chorioretinopathy: results of an international consensus conference. Am J Ophthalmol 2006;141:185-7.

3 Levinson RD, Gonzales GR. Birdshot retinochoroidopathy: immunopathogenesis, evaluation and treatment. Ophthalmol Clin N Am 2002;15:343-50.

4 Shah KH, Levinson RD, Goldhardt R, et al. Birdshot chorioretinopathy. Surv Ophthalmol 2005;50:519-41.

5 Vitale AT, Rodriquez A, Foster CS. Low-dose cyclosporine therapy in the treatment of birdshot retinochoroidopathy. Ophthalmology 1994;101:822-31.

6 Becker MD, Wertheim MS, Smith JR, et al. Long-term follow-up of patients with birdshot retinochoroidopathy treated with systemic immunosuppression. Ocul Immunol Inflamm 2005:13:289-93.

7 Kiss S, Ahmed M, Letko E, et al. Long-term follow-up of patients with birdshot retinochoroidopathy treated with corticosteroid-sparing systemic immunomodulatory therapy. Ophthalmology 2005;112:1066-71.

8 Allison AC, Eugui EM. Immunosuppressive and other effects of mycophenolic acid and an ester prodrug, mycophenolate mofetil. Immunol Rev 1993;136:5-28.

9 Thorne JE, Jabs DA, Qazi FA, et al. Mycophenolate mofetil therapy for inflammatory eye disease. Ophthalmology 2005;112:1472-7.

10 Teoh SC, Hogan AC, Dick AD, et al. Mycophenolate mofetil for the treatment of uveitis. Am J Ophthalmol 2008;146:752-60.
11 Pleyer U, Ruokonen P, Schmidt N, et al. Mycophenolic acid in ocular autoimmune disorders—can we optimise the therapy? Klin Monbl Augenheilkd 2008;225: 66-9.

12 Deuter CME, Doycheva D, Stuebiger N, et al. Mycophenolate sodium for immunosuppressive treatment in uveitis. Ocul Immunol Inflamm 2009;17: 415-19.

13 Doycheva D, Zierhut $M$, Blumenstock $G$, et al. Long-term results of therapy with mycophenolate mofetil in chronic non-infectious uveitis. Graefes Arch Clin Exp Ophthalmol 2011;249:1235-43.

14 Forooghian F, Gulati N, Jabs DA. Restoration of retinal architecture following systemic immunosuppression in birdshot chorioretinopathy. Ocul Immunol Inflamm 2010:18:470-1.

15 Tomkins-Netzer O, Taylor SRJ, Lightman S. Long-term clinical and anatomic outcome of birdshot chorioretinopathy. JAMA Ophthalmol 2014;132:57-62.

16 Nussenblatt RB, Palestine AG, Chan CC, et al. Standardization of vitreal inflammatory activity in intermediate and posterior uveitis. Ophthalmology 1985;92:467-71.

17 Jabs DA, Nussenblatt RB, Rosenbaum JT; Standardization of Uveitis Nomenclature Working Group. Standardization of nomenclature for reporting clinical data. Results of the first international workshop. Am J Ophthalmol 2005;140:509-16.

18 Marmor MF, Fulton AB, Holder GE, et al. ISCEV Standard for full-field clinical electroretinography (2008 update). Doc Ophthalmol 2009;118:69-77.

19 Rothova A, Ossewaarde-van Norel A, Los LI, et al. Efficacy of low-dose methotrexate treatment in birdshot chorioretinopathy. Retina 2011;31:1150-5.

20 Cervantes-Castaneda RA, Gonzalez-Gonzalez LA, Cordero-Coma M, et al. Combined therapy with cyclosporine $A$ and mycophenolate mofetil for the treatment of birdshot retinochoroidopathy: a 12-month follow-up. Br J Ophthalmol 2013;97:637-43.

21 Behrend M. Adverse gastrointestinal effects of mycophenolate mofetil: aetiology, incidence and management. Drug Saf 2001;24:645-63.

22 Doycheva D, Zierhut M, Blumenstock G, et al. Mycophenolate mofetil in the therapy of uveitis macular edema-long-term results. Ocul Immunol Inflamm 2012:20:203-11.

23 Sobrin L, Lam BL, Feuer WJ, et al. Electroretinographic monitoring in birdshot chorioretinopathy. Am J Ophthalmol 2005;140:52-64.

24 Comander J, Loewenstein J, Sobrin L. Diagnostic testing and disease monitoring in birdshot chorioretinopathy. Semin Ophthalmol 2011;26:329-36. 


\section{Mycophenolic acid in the treatment of birdshot chorioretinopathy: long-term follow-up}

Deshka Doycheva, Herbert Jägle, Manfred Zierhut, Christoph Deuter, Gunnar Blumenstock, Ulrich Schiefer, Katarina Stingl, Kai Januschowski, Bogomil Voykov and Nicole Stuebiger

Br J Ophthalmol 2015 99: 87-91 originally published online July 24, 2014 doi: 10.1136/bjophthalmol-2014-305535

Updated information and services can be found at:

http://bjo.bmj.com/content/99/1/87

\section{Notes}

\section{These include:}

References This article cites 24 articles, 1 of which you can access for free at: http://bjo.bmj.com/content/99/1/87\#BIBL

Email alerting Receive free email alerts when new articles cite this article. Sign up in the service box at the top right corner of the online article.

To request permissions go to:

http://group.bmj.com/group/rights-licensing/permissions

To order reprints go to:

http://journals.bmj.com/cgi/reprintform

To subscribe to BMJ go to:

http://group.bmj.com/subscribe/ 